\title{
Encoding variability, levels of processing, and the effects of spacing of repetitions upon judgments of frequency
}

\author{
ROBERT J. ROSE \\ Memorial University of Newfoundland, St. John's, Newfoundland A1B 3X9, Canada
}

\begin{abstract}
Two experiments were carried out to compare the component-levels theory and the levelsof-processing hypothesis as explanations of the effect the spacing between repetitions of an item has upon the retention of that item. Retention was measured by judgments of frequency, frequency discrimination, and derived recognition scores. Variable encoding contexts facilitated recognition relative to repeated encoding contexts, while the latter biased subjects toward giving high judgments of frequency. The results in general were consonant with predictions derived from a levels-of-processing explanation of the spacing effect rather than the componentlevels theory. A modification of the latter theory to incorporate the former was suggested as a view of the memorial representation of repeated stimuli.
\end{abstract}

The term "spacing effect," also known as the "lag effect," refers to the empirical fact that memory for repeated items improves up to a point as the interval (i.e., the spacing or lag) between the repetitions increases. The spacing is usually measured in terms of the number of other items intervening between the repetitions, although some investigators have measured the spacing in units of time (e.g., Hintzman $\&$ Rogers, 1973). The facilitation of the retention of repeated items by increased spacing is exceedingly general and robust, having been found with a variety of materials and for several measures of retention. However, explanations of the spacing effect have been less robust than the phenomenon itself (see the reviews by Hintzman, 1974, 1976), and the experiments reported here address themselves to this explanatory problem.

Glenberg (1979) has postulated a very thoughtful component-levels theory to account for the effects of spacing, a view that is an expansion of the encoding variability hypothesis put forward earlier (Glenberg, 1976). The theory's first basic principle is that "a repetition is potentially effective to the degree that the second presentation allows for the storage of information distinct from that stored at the first presentation." Furthermore, the theory envisages a memory trace as a store of information in the form of attributes or components, of which there are three types: contextual, structural, and descriptive. Contextual components include such information as the time of an event, the physical environment in

This research is based on a $\mathrm{PhD}$ thesis submitted to the Department of Psychology, Memorial University of Newfoundland, by the author and supervised by E. J. Rowe. Requests for reprints should be sent to Robert J. Rose, Department of Psychology, Memorial University of Newfoundland, St. John's, Newfoundland A1B 3X9, Canada. which an event occurs, and the learner's cognitive and affective state. Structural components reflect the structure the learner imposes upon the events occurring within the learning situation (i.e., which items are associated, categorized, or chunked together). Descriptive components constitute the properties of an individual event (i.e., its orthography, articulation, and meaning). These components reflect the semantic memory representation of the event in its current context.

Glenberg (1979) argues that the typical spacing effect, as depicted schematically by the curve $A B$ in Figure 1, occurs because the traces of an item repeated at long lags show more variability among their three types of components than the traces of an item repeated at short lags. Hence, according to the first basic principle of component-levels theory, repetitions will be potentially more effective for memory

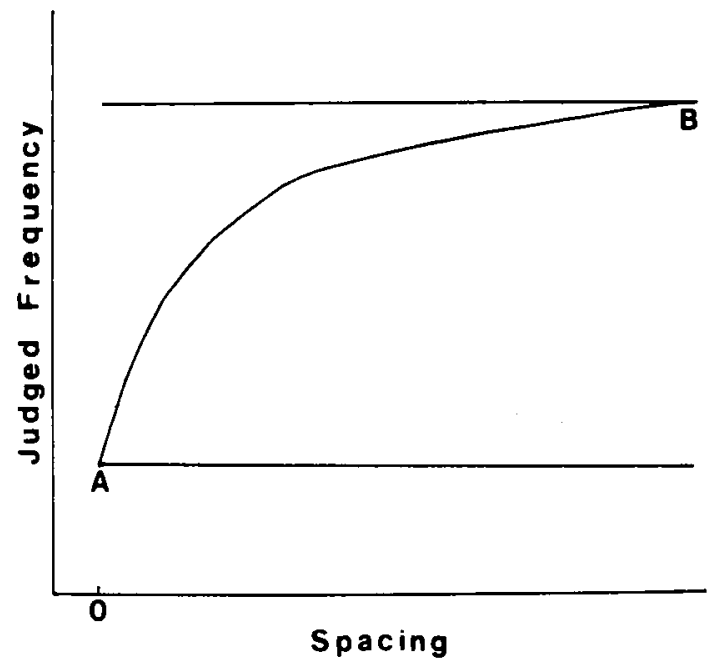

Figure 1. Hypothetical spacing functions predicted for Experiment 1 (see text for explanation). 
at long lags because of the differential encoding and storage of their components. Two predictions follow directly from this view. One is that forcing subjects to encode each repetition of an item in a different context should attenuate the effect of spacing and produce a high level of retention, as shown by the horizontal line through $\mathbf{B}$ in Figure 1. This pattern of results should emerge since all repeated items, regardless of spacing, should show differential encoding among a number of components. The second prediction is the converse of the first; namely, forcing subjects to encode every presentation of a repeated item in the same context should also eliminate the spacing effect but at a low level of retention, as depicted by the horizontal line through $\mathbf{A}$.

In order to test the predictions derived from the component-levels theory, the subjects in the experiments reported here were asked binary questions of a semantic nature concerning the items presented on a study trial. Such questions, requiring as answers "yes" or "no," have been used before in incidental learning paradigms (see Craik \& Tulving, 1975, for example). Furthermore, in one condition, a different question was asked for each item in the sequence, whether it was a repetition or not. In a second condition, the same binary question was asked for each presentation of the same item, but different questions were asked of different items. The question condition, different or same, was designed to manipulate the cognitive context within which each repeated word was processed; that is, contextual and, to a certain extent, descriptive components were assumed to be affected.

Strictly speaking, of course, one cannot have complete experimental control over encoding contexts. If temporal factors were important components of the encoding context, then a spacing effect could still occur, since temporal differences are obviously greater among items repeated at long lags than among items repeated at short lags. However, these temporal differences do not appear to be salient attributes of memory. For instance, Hintzman and Block (1973) and Hintzman, Summers, and Block (1975) found that subjects could judge to a significant degree of accuracy the spacing between two presentations of a single word or between the presentations of two related words. The subjects could not judge, on the other hand, the spacing between two unrelated words, even though the temporal differences were the same as for the other two types of experimental items. One seems justified, then, in predicting from component-levels theory that forcing upon each presentation of an item differential or repeated encoding would at least approximate the straight lines in Figure 1 through B and A, respectively.

An alternative to the component-levels theory as an explanation of the spacing effect is a levels-of- processing view, as suggested by Rose and Rowe (1976) and elaborated by Lockhart, Craik, and Jacoby (1976). Briefly, the explanation states that, when an item is repeated in a study sequence, the subject attempts to contact the memory trace of the first presentation. When repetitions are close together, this contact is made relatively easily by scanning recent episodic memory. As the spacing between repetitions increases, more effort than mere scanning must be employed, and the subject must then reconstruct something approaching the encoding of the original event. This reconstruction process involves a deeper level of processing than the scanning process and therefore leads to better long-term retention. According to this view, then, the necessary condition for good retention is not variable encoding but encoding to a deep level.

It should be pointed out that the levels-of-processing view and component-levels theory share a number of similarities. Both approaches view a memory trace as a bundle of components that include the nature of the corresponding stimulus event, the type of processing task, the context of the event, the subject's strategies and expectations, and so on. There appears, however, to be at least one basic difference. Glenberg $(1976,1979)$ conceives of the trace of a repetition as "overlapping" the trace of the initial presentation of the event. As the spacing between repetitions increases, this overlapping is less and less, so that the traces of the repetitions contain considerable variability among their components, including presumably the cognitive encoding of the repetitions. Lockhart et al. (1976) suggest the exact opposite for repetitions at longer spacings. Here, the scanning procedure cannot be used efficiently so that contact between the current repetition and the trace of the initial presentation occurs via the aforementioned reconstructive process. In their view, the encoding of the repetition is guided, not only by the representation of the stimulus event in semantic memory, but also by previous encodings of the event in the study sequence. If the previous presentations have occurred at fairly long spacings, then they will be approximate reconstructions of the first presentation. Assuming that this first presentation was processed to a deep level (as should be the case when a semantic task is required), all repetitions of the event will be processed to a deep level. To quote from Jacoby (1974), the reconstructive process "is much like a servomechanism with constructive activities ... homing in on the trace of the target item." Thus, according to the levels-ofprocessing view, as the spacing between repetitions increases, the encodings of these repetitions become, if anything, more similar and not less so. This view pertains at least to the type of cognitive processing carried out on the repeated event.

Applying the levels-of-processing arguments to 
Figure 1, one would predict along with the componentlevels theory that the different-question condition of Experiment 1 will produce results approximating the straight line through B. This outcome should occur, however, because subjects under this condition will not be able to scan recent memory for an answer but will have to process each question separately to a deep level, regardless of the spacing between repetitions. This difference between the two hypotheses lies in the prediction of the outcome for the same-question condition. The component-levels theory predicts that imposing the same encoding context on all presentations of a word should attenuate the effect of spacing and produce a relatively low level of retention, as shown by the straight line through $\mathrm{A}$. The levels-ofprocessing hypothesis, on the other hand, leads one to expect the usual spacing function, as depicted by the curve $A B$. This outcome follows from a shallow scanning of recent memory for the response when answering a question repeated after a short lag and a deep reconstruction of the original cognitive process when answering a question repeated after a long lag.

The encoding variability and levels-of-processing hypotheses were compared as explanations of the spacing effect in two experiments in which retention was measured by judgments of frequency. Judgments of frequency were used because they are a direct measure of memory for repetitions and also allow one to derive measures of recognition. In addition, Glenberg $(1976,1979)$ has dealt quite extensively with the effect of spacing upon recall and recognition but not upon judgments of frequency. (He does suggest parenthetically, however, that contextual components may be involved in forming these judgments.)

To date, no clear explanation of the process involved in forming judgments of frequency has emerged, although subjects must presumably attempt to contact all traces of an item presented within the experimental context. Howell (1973) reviewed the hypothetical representations of event frequency in memory and concluded that most evidence favored either a multiple-trace or a multiple-process view, which for measures of episodic memory are identical. This being the case, there seem to be two basic ways in which a subject can use the test item to judge its frequency of occurrence. The test item could contact as few as one of the traces of the repeated item, and this trace could be used as a retrieval cue for the other traces. The component-levels theory would appear to be incompatible with this form of retrieval. As mentioned above, the theory predicts that a retrieval cue is efficient to the extent that it has components in common with one or more traces. However, the commonality of components, especially contextual components, supposedly decreases over spacing. Hence, the component-levels theory as applied here would predict a decrease in judgment of frequency over spacing and not the typical increase.

The second way to judge the frequency of occurrence of a test item within the study sequence would involve contact between the encoding of the test item and each of the traces of its presentations within the sequence. Here, the component-levels theory would find no difficulty of prediction. In the typical case, in which the test item is presented after a rather long retention interval, the components of the test item would share a small degree of commonality with the components of each trace, but with equal probability. Now, if the presentations within the sequence were close together, their traces would share many common components. Hence, although the subject may successfully contact these components, he would have difficulty in deciding which components belong to which trace and in judging how many "bundles" of components there were. At higher values of spacing, on the other hand, various traces would be more distinct, and the subject would then give higher judgments of frequency.

The levels-of-processing view would have no difficulty in accounting for the spacing effect within either of the two suggested processes for forming judgments of frequency. Since the encoding of a repetition after a fairly long interval is a reconstruction of the initial encoding, the trace of the repetition can function perfectly well as a cue in accordance with the principle of encoding specificity (Tulving, 1976) to produce the evidential increase in retention over spacing. If judgments of frequency are formed in the second manner suggested above, that is, via contact between the test item and each individual trace, then judgments should still increase over spacing because the traces of spaced repetitions are the products of encoding to a deep level. As such, they are deemed by Lockhart et al. (1976) to be relatively distinctive and hence easily accessed by a test item. A question arises as to how the traces of items repeated at long lags can be both distinctive and similarly encoded. The final discussion will return to this question. Suffice it for now to claim that the arguments of the levels-of-processing approach satisfy the effect of spacing upon judgments of frequency formed by either of the two suggested processes.

To summarize, two experiments were carried out in which subjects were asked a simple question concerning each word in the experimental sequence. Under one condition, every item in the sequence, whether a repetition or not, was asked a unique question. In a second condition, all presentations of the same stimulus were accompanied by the same question. Following the question task, subjects were asked to judge the frequency of occurrence of a 
number of words in the sequence. With reference to Figure 1, the component-levels theory predicts that the different-question condition will exceed the samequestion condition considerably, that there will be little or no overall effect of spacing, and that no significant Question Condition by Spacing interaction will emerge. The levels-of-processing hypothesis predicts that the different-question condition will exceed the same-question condition only at short spacings, that there will be an effect of spacing (due to the same-question condition, mainly), and that there will be a strong Question Condition by Spacing interaction.

\section{EXPERIMENT 1}

\section{Method}

Materials. The sequence items were common words (A or AA by the Thorndike-Lorge, 1944, word count) of one or two syllables and five or six letters each, except for the seven-letter word, "college." Each subject was given a pack of cards, each of which contained a word typed in capital letters with a simple binary question (i.e., to be answered "yes" or "no") pertaining to that word typed in lowercase beneath it. The questions used in the sequence all referred to some semantic aspect of the words they accompanied (e.g., BUTTER Is this a dairy product?). Words appeared in the pack two, three, or five times at spacings of $0,1,2,4,8$, or 16 with 27 filler items appearing once each. Four words were assigned to each frequency by spacing cell, which produced 267 cards per pack.

The same-question and different-question conditions were employed as a between-subjects variable. For each condition, six packs of cards were constructed in order to counterbalance specific item effects across frequencies and spacings. Thus, each experimental word appeared once at each level of spacing and twice at each presentation frequency. Within the same-question condition, the same binary question was asked for each presentation of the same item, but different questions were asked of different items. This procedure was intended to reduce the tendency to form associations among different items but not to force subjects to process the various presentations of the same item differentially. Within the different-question condition, a different question was asked for each item in the sequence, whether it was a repetition or not. Furthermore, the questions associated with each of the repetitions in this condition biased the same meaning of a repeated word. For example, the questions accompanying the word "earth" all concerned "earth" as a planet, not "earth" as soil, and so on. In this way, the verbal contexts were considered to differ under the different-question condition mainly in a nonsemantic sense. More will be made of this point in the final discussion.

The word sequences within each of the two groups of packs were identical, although the accompanying question conditions were different. For the same-question condition, half of the questions required positive answers and half required negative answers. The order of the positive and negative questions was counterbalanced throughout these packs in order to avoid long sequences containing only one type of question. Under the differentquestion condition, positive and negative questions were counterbalanced within the repetitions of a particular word. For example, words appearing five times were accompanied by three positive and two negative questions in random order. Filler words, of course, could not be assigned to one question condition or another. Rather, half of them were accompanied by a question requiring a positive answer, while the other half required a negative answer.

Subjects. The subjects were 48 undergraduates of Memorial University who were assigned randomly to either the differentquestion group or the same-question group. Four subjects were assigned to each of the six packs of cards within each group. The testing was carried out in small groups of six or fewer subjects. Each subject was paid $\$ 3$ for participating.

Procedure. The subjects were given an instruction sheet that informed them that the experiment was concerned with people's view of a number of common concepts. Accordingly, they would be required to answer "yes" or "no" to a number of simple questions relating to these concepts. The subjects in the samequestion group were told that some questions were repeated as a check for consistency in their answers. Following 12 words of practice, the subjects went through the pack of cards at their own pace and wrote the answer to each question in the appropriate space on a response sheet. Half of the subjects went through the pack in reverse order.

When they had finished the question task, the subjects were given an unexpected test requiring them to judge how often each of the words on the test sheet had appeared in the pack of cards. The test consisted of all 72 experimental words plus 14 new words chosen from the experimental population and 14 filler items. The fillers were randomly chosen from those once-presented words that did not occupy primacy or recency positions in the pack. The judgments of frequency test were unpaced. The entire experiment required about $40 \mathrm{~min}$.

\section{Results and Discussion}

Judgments of frequency. The mean judgment of frequency as a function of question condition, presentation frequency, and spacing is shown in Figure 2. It can be seen that the form of the results at each level of frequency resembles the predictions derived from the levels-of-processing hypothesis rather than the component-levels theory; that is, the spacing effect was attenuated under the differentquestion condition but not under the same-question condition. A result unpredicted from Figure 1 was the tendency for judgments under the same-question condition to exceed generally the judgments under the different-question condition. This tendency has been found in similar situations (Hintzman \& Stern, 1978; Rowe, 1973a, 1973b) and will be discussed further in a later section of the results.

These appearances were confirmed by analyses of

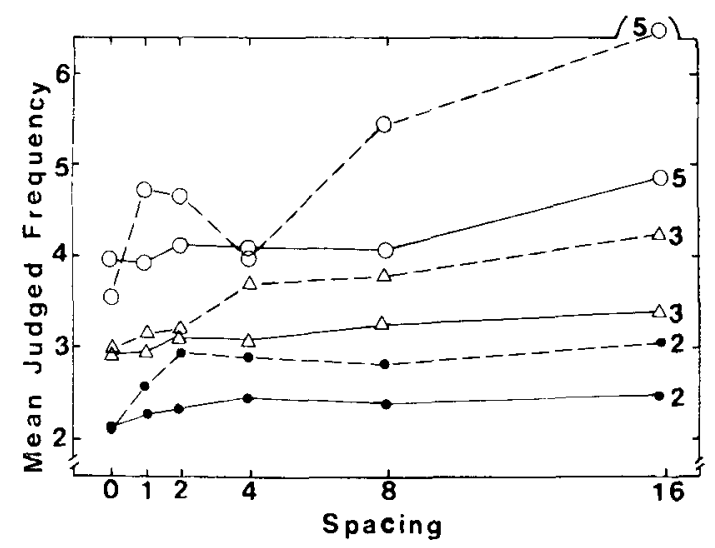

Figure 2. Mean judgment of frequency in Experiment 1 as a function of question condition, presentation frequency (two, three, five), and spacing. The different-question condition is represented hy the solid lines. 
variance. A three-factor analysis showed significant effects of question condition $[F(1,46)=4.75, p<.05$, MSe $=10.10]$ and of spacing $[F(5,230)=24.12$, MSe $=.92]$ and a significant Spacing by Question Condition interaction $[\mathrm{F}(5,230)=6.32$, $\mathrm{MSe}=.92]$. (In all analyses reported in this paper, the value of $\mathrm{p}<.001$ unless stated otherwise.) Because the triple interaction was significant $[\mathrm{F}(5,460)=3.02, \mathrm{p}<.01$, $\mathrm{MSe}=.73]$, further analyses were carried out for the different-question and same-question conditions separately. These showed for the different-question condition a significant effect of spacing $[F(5,230)=$ $3.37, \mathrm{p}<.01]$ but no Frequency by Spacing interaction. The effect of spacing was due to judgments of frequency at a spacing of 16 exceeding those at spacings of 0 and 1 ( $p<.05$, according to Tukey's $b$ test). For the same-question condition, there was, among other things, a significant Frequency by Spacing interaction $[\mathrm{F}(10,460)=7.92, \mathrm{MSe}=.73]$. One-factor analyses showed that spacing was significant under the same-question condition at each level of presentation frequency $[\mathrm{Fs}(5,460)>4.08$, ps $<$ $.01 \mathrm{~J}$, with the effect increasing over presentation frequency.

Judgments of frequency were also examined for positive and negative questions separately under the same-question condition. This examination was carried out because Craik and Tulving (1975) have shown that long-term recall is often better when positive questions are asked in an incidental learning task than when negative questions are asked. An analysis of these data showed that judgments of frequency were indeed generally higher for words asked positive questions than for words asked negative questions. However, there were no significant interactions involving type of question asked, and hence this factor had no confounding effect for the purposes of this experiment. In addition, the incidental tasks were scored, and the judgments by question type (i.e., positive vs. negative) were adjusted according to the answers given by the subjects. The error rates were $4.67 \%$ for the different-question condition and $5.06 \%$ for the same-question condition. The adjustments to the judgments made little difference to the cell means (i.e., the positive and negative errors tended to cancel out). For this reason, further analyses were not carried out in this area.

Finally, judgments of frequency for words presented once were compared with judgments for words presented with massed repetition under the same-question condition. This comparison arose from Glenberg (1976), who states that a repetition of an item at very short lags will produce no learning beyond that produced by a single presentation. This view is further implied by the component-levels theory, at least for a situation such as the same-question condition, and by Craik and Lockhart (1972). An analysis of variance showed a significant difference among the means $[F(3,69)=17.85$, MSe $=.78]$. The mean for once-presented words, which was 1.88 , did not differ from that of massed words at frequency of two, but all other pairs were significantly different $(p<.01)$. These results are inconsistent with the positions of Craik and Lockhart (1972) and Glenberg $(1976,1979)$, unless one argues that these positions do not apply to massed presentations whose frequency exceeds two.

Recognition scores. Derived recognition scores were calculated by considering that any experimental word given a judgment of zero was a "miss" and all other judgments of an experimental word were hits. The probability of correct recognition is shown by conditions collapsed across frequency in Table 1. It can be seen that recognition was consistently higher for the words in the different-question condition than for those in the same-question condition. A threefactor analysis of variance of recognition misses confirmed that question type had a significant effect $[\mathrm{F}(1,46)=17.17, \mathrm{MSe}=.50]$. No other factor had a significant effect.

Recognition false alarms for the two groups were calculated and analyzed. The different-question group had a higher rate (.17) than the same-question group (.10), but the difference was not significant [t(46 = $1.73, .10>\mathrm{p}>.05, \sigma_{\mathrm{est}}=3.79$ ].

A further check of response bias was carried out by correcting the recognition hits of each subject for guessing through the use of the formula: corrected $P_{H}=\left(P_{H}-P_{F / A}\right) /\left(1-P_{F / A}\right)$, where $P_{H}$ is the proportion of hits and $P_{F / A}$ is the proportion of false alarms. Since false alarms cannot be attributed to various levels of spacing, the same value of $P_{F / A}$ (.10 or .17) was applied to each frequency lag cell within each question condition. The mean difference between the corrected and uncorrected hit rates was less than .01 for all cells. For the same-question condition, the overall mean probability of correct recognition remained unchanged by correction at $\mathbf{9 3 2}$. For the different-question condition, the corrected overall hit rate was slightly below the uncorrected overall hit rate (.985 vs. .987). An analysis of variance of the

Table 1

Mean Probabilities of Correct Recognition in Experiments 1 and 2 for Each Spacing and Question Condition

\begin{tabular}{lcccccc}
\hline & \multicolumn{6}{c}{ Spacing } \\
\cline { 2 - 7 } Question \\
\cline { 2 - 7 } & \multicolumn{7}{c}{ Condition } & 0 & 1 & 2 & 4 & 8 & 16 \\
\hline & & \multicolumn{5}{c}{ Experiment 1 } \\
Different & .983 & .983 & .990 & .990 & .990 & .987 \\
Same & .917 & .941 & .917 & .917 & .944 & .955 \\
& & & Experiment 2 & & \\
Different & .997 & .973 & .987 & .980 & .990 & .973 \\
Same & .937 & .940 & .930 & .960 & .967 & .957 \\
\hline
\end{tabular}


corrected recognition measures was deemed unnecessary. Hence, the hit rates appear to be free of any significant effect of differential response bias. More important, the tendency for subjects in the samequestion condition to give higher judgments of frequency does not arise from their differential bias toward recognition false alarms, as one might expect if judgments of frequency and recognition reflect the same underlying process (see Underwood, Zimmerman, \& Freund, 1971).

The recognition results for the same-question condition were also examined for positive vs. negative questions. The negative questions produced 72 misses (hit rate of .92), compared with only 36 misses (hit rate of .96) for the positive questions $[F(1,46)=$ 15.41, MSe $=.05]$. However, since no other factor or interaction had a significant effect, the effect of type of question upon recognition was not crucial.

Judgments of frequency conditional upon correct recognition were calculated for each question condition by frequency spacing cell. These yielded patterns that were very similar to those depicted in Figure 2, except that the judgments for the samequestion condition tended to be slightly higher relative to the judgments for the different-question condition. Hence, the Question Condition by Spacing interaction did not appear to reflect any differential effect of recognition upon the two types of encoding context.

Two further points should be made in concluding this section. One is that the recognition hits are undoubtedly affected by a ceiling, since it is unusual to find no effect of presentation frequency over a range of two to five. The spacing factor, which generally exerts a considerably weaker effect than presentation frequency, would therefore be affected by the ceiling also. As a result, the effect of spacing within the same-question condition is merely suggestive. Thus, the recognition data found here do not contribute significantly to any explanation of the spacing effect, although the pattern of the results conform more to the predictions of the levels-ofprocessing view than to the component-levels theory.

The second point to be made is that the significant results found here are inconsistent with those of Rowe (1973a, 1973b), who found that the verbal context of words had no effect upon their recognition. However, the greatest differences lie at small values of lag, and it is possible that no significant differences would exist if the long lags used by Rowe were also used here. On the other hand, the effect of encoding context upon recognition found here replicates that found by Hintzman and Stern (1978, Experiment 2).

Discrimination coefficients. Up to this point, the data replicate the results found by Hintzman and Stern (1978) as far as the effects of encoding context variability are concerned. Specifically, variable encoding contexts, as opposed to repeated encoding contexts, lead to increased probability of correct recognition and lower judgments of frequency on a test of retention that follows the study trial. To explain this pattern of results parsimoniously, one can assume that variable encoding of $n$ repetitions of an item lays down $\mathrm{n}$ distinctive traces, any one of which is relatively easy to contact in a subsequent test of retention. Therefore, variable encoding enhances recognition. However, judgments of frequency of an item require the retrieval of all $\mathbf{n}$ traces, and this type of retrieval is inhibited if these traces are distinct (i.e., if they share relatively few common attributes). Therefore, variable encoding contexts lead to low judgments of frequency. Hintzman and Stern support the same basic position regarding judgments of frequency when they say that "the more similar were two past events, the more likely it is that remembering one of them will remind you of the other" $(1978$, p. 547).

If this view is correct, then one may infer that discrimination of the various levels of presentation frequency should be better under the same-question condition. This inference follows because subjects under the same-question condition will be able to retrieve all of the memory traces of a repeated item more efficiently, at least at higher values of spacing, than subjects under the variable-question condition. Under variable encoding conditions, subjects are more likely to "miss" some traces, and hence they do worse on a measure of frequency discrimination. In order to test this argument, discrimination coefficients (Flexser \& Bower, 1975) involving the experimental words were calculated for each subject at each level of spacing. These coefficients are indices of the (Pearson product-moment) correlation between true frequency and judged frequency; they are shown in Table 2 for each group. These results show that the discrimination coefficients are in fact generally lower for subjects under the same-question condition than for subjects under the different-question condition. This difference was found to be significant by an analysis of variance $[F(1,46)=4.52, p<.05$, $\mathrm{MSe}=.09]$. Furthermore, there was a significant Question Condition by Spacing interaction $[F(1,230)=$ $3.24, \mathrm{p}<.01, \mathrm{MSe}=.05]$. Separate analyses of the

\section{Table 2}

Mean Discrimination Coefficients in Experiments 1 and 2 for Each Spacing and Question Condition

\begin{tabular}{lcccccc}
\hline & \multicolumn{6}{c}{ Spacing } \\
\cline { 2 - 8 } $\begin{array}{l}\text { Question } \\
\text { Condition }\end{array}$ & 0 & 1 & 2 & 4 & 8 & 16 \\
\hline & & \multicolumn{5}{c}{ Experiment 1} \\
Different & .451 & .499 & .504 & .482 & .446 & .604 \\
Same & .280 & .491 & .439 & .237 & .512 & .570 \\
& & & Experiment 2 & & \\
Different & .455 & .500 & .490 & .526 & .597 & .480 \\
Same & .544 & .363 & .492 & .435 & .589 & .537 \\
\hline
\end{tabular}


results for each question condition showed that the effect of spacing was not significant for the differentquestion condition but was significant for the samequestion condition $[F(5,230)=8.99]$.

The frequency discrimination coefficients, then, do not support the parsimonious explanation of the judgments of frequency and recognition data given above. Rather, they support the contention that variable encoding contexts enhance retention in general, while repeated contexts somehow bias subjects toward giving high judgments of frequency. This bias, however, does not arise from a tendency to produce recognition false alarms. Moreover, the pattern of the discrimination data resembles the pattern predicted by the levels-of-processing hypothesis in Figure 1. In other words, there is a "typical" spacing effect with the same-question condition, an attenuated spacing effect with the different-question condition, and the level of retention with repeated encoding contexts is lower than the level with variable encoding contexts, especially at short spacings.

The next experiment was carried out to test the generality of the findings of Experiment 1 . Here, the question conditions were applied to separate groups, and some confounding of encoding context with individual differences between the subjects in the two groups may have occurred. A check of this possible confounding was carried out in Experiment 2 by repeating the basic procedure but introducing the question condition as a within-subjects factor.

\section{EXPERIMENT 2}

\section{Method}

Materials and Procedure. The procedure and judgment of frequency test used here were the same as those used in Experiment 1 . The materials were also the same, except that, within each pack, half of the experimental words were accompanied by a different question on each presentation of the word, while half were accompanied by identical questions on each presentation of the same word. For this experiment, only six packs of cards were required, not two groups of six. Within the different-question condition, half of the questions were positive and half were negative. For the two words appearing three times each in a frequency by spacing cell in the different-question condition, one word had one negative and two positive questions and the second word had one positive and two negative questions. For the two words appearing five times in a cell under the different-question condition, the split was $3: 2$ and $2: 3$. In these conditions, the positive and negative questions occupied alternate ordinal positions. For the two words appearing in each frequency by spacing cell under the same-question condition, one word was accompanied on all presentations by the same positive question, while the second word was always accompanied by the same negative question. Again, half of the filler words were accompanied by a question requiring a positive answer and the other half required a negative answer.

Subjects. The subjects were 48 undergraduates of Memorial University who were paid $\$ 3$ each for their participation. Eight subjects were assigned to each of the six packs of cards. The testing was carried out in small groups of not more than six subjects each.

\section{Results and Discussion}

Judgments of frequency. The mean judgment of frequency for each frequency by spacing cell within each condition is depicted in Figure 3. The initial analysis of variance involved the factors of presentation frequency, spacing, and question condition. The analysis showed the usual strong effect of frequency $[F(2,94)=173.29, \mathrm{MSe}=3.72]$ and a significant effect of spacing $[\mathrm{F}(5,235)=21.27, \mathrm{MSe}=2.27]$. There was no effect of question condition $(F<1)$, but there were significant interactions involving the factor of question condition. The two important interactions were Question Condition by Spacing $[\mathrm{F}(5,235)=8.42, \mathrm{MSe}=2.15]$, which reflected the greater effect of spacing under the same-question condition relative to the different-question condition and the triple interaction $[\mathrm{F}(10,470)=2.15, \mathrm{p}<.025$, $\mathrm{MSe}=1.53]$.

The significant triple interaction indicated different frequency by spacing patterns for the two question conditions. Separate analyses of the judgments for each type of question condition were carried out. For the different-question condition, spacing had a significant effect $[F(5,235)=2.66, p=.025]$, but there was no significant Frequency by Spacing interaction. A test of the individual means showed that the judgments at a spacing of eight significantly exceeded the judgments at spacings zero and one.

For the same-question condition, the most interesting result from the analysis was a significant Spacing by Frequency interaction $[F(10,470)=4.46]$. Further analyses found a significant effect of spacing at each level of frequency $[\mathrm{Fs}(5,470)>8.47]$. The comparison of individual means showed only a massed-presentations effect at a frequency of 2, while at Frequency Level 5 the judgments at spacings of 8 and 16 exceeded all others. At a frequency of three, no level of spacing differed from the adjacent

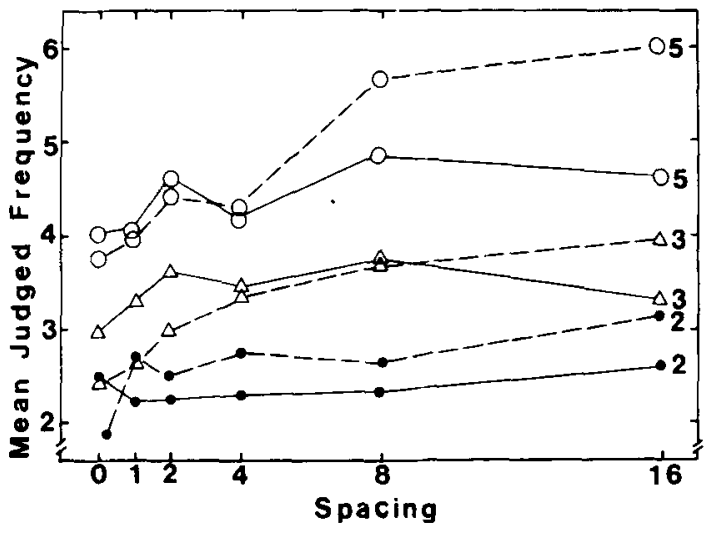

Figure 3. Mean judgment of frequency in Experiment 2 as a function of question condition, presentation frequency (two, three, five), and spacing. The different-question condition is represented by the solid lines. 
level, but all other pairs of comparisons were significantly different.

As in Experiment 1, the judgments of frequency for positive and negative questions within the samequestion condition were analyzed. As before, there were no significant interactions with this factor. Unexpectedly, the mean judgment for words asked positive questions (3.54) was not significantly different from the mean judgment following negative questions (3.46). The judgments of frequency of once-presented words were again compared with the judgments of massed repetitions. As before, the mean for the once-presented words (1.54) did not differ from the mean judgment of two massed presentations, but all other comparisons were significant $(\mathrm{p}<.01)$.

Recognition scores. Derived recognition scores were calculated as in Experiment 1. The probability of correct recognition is shown by conditions collapsed across frequency in Table 1. It can be seen that recognition was consistently higher for words in the different-question condition than for those in the same-question condition. An analysis of variance of recognition misses confirmed that question type had a significant effect $[F(1,47)=19.81, \mathrm{MSe}=.11]$. There were no other significant effects except for presentation frequency $[\mathrm{F}(2,94)=5.81, \mathrm{p}<.01$, MSe $=.06]$. Unfortunately, false alarms could not be categorized separately for the two question conditions due to the within-subjects application of that factor. The false alarm rate here was .20, compared with a mean rate of .14 for Experiment 1 .

The recognition results for the same-question condition were examined for positive vs. negative questions. The negative questions produced 66 misses, while the positive questions produced only 27 misses $[\mathrm{F}(1,47)=18.38, \mathrm{MSe}=.06]$. As in Experiment 1 , no other factor or interaction was significant. Judgments of frequency conditional upon correct recognition were once again calculated and examined. As before, these yielded patterns very similar to those found with the unconditional judgments.

Discrimination coefficients. The discrimination coefficients for each spacing and question condition are shown in Table 2. The data for the same-question condition are not as consistent as those in Experiment 1 . In particular, the high coefficient for a spacing of zero appears to be anomalous (see Rowe \& Rose, 1977). The inconsistency may arise in part from the small amount of data entering the correlation at each spacing for each subject, specifically, six pairs of numbers (compared with 12 pairs in Experiment 1). An analysis of variance showed that only spacing had a significant effect $[F(5,235)=2.39$, $\mathrm{p}<.05$, MSe $=.12$ ]. However, insofar as the samequestion condition did not exceed the different-question condition on the discrimination coefficients, the results here support those found in Experiment 1 and militate against the view that multiple traces are easier to retrieve if those traces have been encoded within repeated contexts.

The purpose of Experiment 2 was to check the extent to which the results of Experiment 1 could be attributed to its between-subjects paradigm. The results of Experiment 2 indicate that the findings of Experiment 1 apply to within-subjects paradigms as well. Specifically, the levels-of-processing hypothesis accounts for the spacing effect better than the component-levels theory, and variable encoding contexts enhance the retention of repeated items better than repeated encoding contexts.

\section{GENERAL DISCUSSION}

The major purpose of the preceding experiments was to test predictions derived from the componentlevels and levels-of-processing hypotheses as explanations of the spacing effect. To reiterate a point made in the introduction, the levels-of-processing and component-levels approaches have much in common but differ fundamentally in their conception of the trace of a repeated item. The component-levels theory envisages the trace of a repetition as increasingly dissimilar from the trace of the original presentation as spacing increases. On the other hand, the levelsof-processing hypothesis postulates that the trace of a repetition after a fairly long lag is in fact something like a replica of the original trace, at least as far as the cognitive processing is concerned. Furthermore, it follows from the component-levels theory that enforced similar encoding and enforced differential encoding of repetitions would lead to results approximated by the straight lines through $\mathrm{A}$ and $\mathrm{B}$, respectively, in Figure 1. The levels-of-processing hypothesis, however, indicates that the same manipulations would produce results approximated by the curve $A B$ and straight line through $B$, respectively. The judgments of frequency and discrimination coefficients of Experiments 1 and 2 were found to be consonant with the levels-of-processing hypothesis but not with the encoding variability hypothesis. The derived recognition hit rates were also consonant with the levels-of-encoding hypothesis insofar as the hit rate for the same-question conditions tended to increase over spacing, although the effect was not significant.

Besides accounting for the results found here, the levels-of-processing hypothesis has two other explanatory advantages. It avoids the logical inconsistency of the component-levels theory when one trace of a repeated item is used as a cue to retrieve other traces of that item. This inconsistency was mentioned in the introduction and arises from the problem of reconciling encoding variability with the principle of 
encoding specificity. Second, the levels-of-processing view implies that the reconstructive process will proceed only if the initial stages of processing a repetition indicate it to be a repetition. This suggests in turn that only correctly recognized repetitions will show a spacing effect and that correctly recognized repetitions will be better retained on a later test. Empirical evidence supports both of these implications (Johnston \& Uhl, 1976; Melton, 1967). On the other hand, the component-levels theory suggests the opposite results, since one would expect that a tendency to recognize a repetition as such would lead to less variability of encoding among repetitions and hence to poorer retention.

A second outcome of these experiments is the effect that variable encoding contexts have upon levels of retention. The pattern found here is further support for the pattern found by Hintzman and Stern (1978). Specifically, variable encoding contexts enhance recognition (and recall as well, according to Hintzman and Stern) but produce lower judgments of frequency than repeated encoding contexts. This pattern indicates that retrieval of all of the traces of a repeated item is relatively easy, given that those traces are similarly encoded and that at least one of them is retrievable. On the other hand, the results with the discrimination coefficients of Experiments 1 and 2 showed that retrievability of multiple traces is at least as efficient following variable encoding contexts as following similar encoding contexts. A parsimonious reconciliation of these results appears to be difficult, and further speculation in this area should await further research. An initial suggestion would be an attempted replication of the discrimination coefficient results through the use of forced-choice judgments, which should also be free of "bias."

Attention must now be turned to some points raised in the introduction. It will be recalled that stress was laid in the Method section of Experiment 1 on the use of questions in the different-question condition that biased the same meaning of a word. A number of earlier studies had tested the interaction between encoding variability and spacing by using homographs whose various meanings were biased by the encoding context. Most of these studies failed to support the predictions derivable from an encoding variability (i.e., component-levels) hypothesis (e.g., D'Agostino \& DeRemer, 1973; Johnston, Coots, \& Flickinger, 1972; Madigan, 1969; Thios, 1972). For this reason, it was felt that a fairer test of the component-levels theory here should involve a procedure that maintained as far as possible the same denotative meaning of a word across its repetitions.

One exception to the failure of homographic stimuli to support the encoding variability hypothesis as an explanation of the spacing effect is the work of Gartman and Johnson (1972). They measured the recall of "critical" words whose meanings were biased by the two words in the sequence that preceded them. In some cases, the biasing words were identical in each of two presentations (e.g., arm, leg, foot; arm, leg, foot); in other cases they biased different meanings of the critical words (e.g., arm, leg, foot; inch, meter, foot). The results for the identical- and different-biasing conditions approximated the straight lines through $A$ and $B$, respectively, in Figure 1 of this paper, with recall under the differentmeaning condition exceeding recall under the samemeaning condition by a factor greater than two. These results, however, are explicable within the levels-of-processing approach, as well as within the component-levels view. When the subject meets the cue words (arm, leg) for the second time, he will expect the word "foot" again. If the expected word appears, it will be processed quickly and superficially in accordance with the usual processing of expected stimuli (see Lockhart et al., 1976, pp. 96-99). Hence, in the identical-biasing condition, the expected critical word is processed to a shallow level, even after a lag of 20 , and it is poorly recalled. Within the differentmeaning condition, the subject is not set to expect "foot" by the novel cues (inch, meter), and so the word must be processed to a deep level again, even after a short lag.

Finally, there remains the major question of the subjects' ability to distinguish the traces of an item repeated at long lags, given that these traces are encoded in a similar fashion according to the levelsof-encoding approach. The aforementioned studies by Hintzman and Block (1973) and Hintzman et al. (1975) appear to be relevant here. To recapitulate, they found that subjects could judge to a significant degree the spacing between two presentations of a word or between the presentations of two related words but not between the presentations of two unrelated words. These results could reflect the encoding of spacing as an attribute of memory for identical or related nominal stimulus events. Alternatively, the results could reflect an interaction between the type of processing carried out on the pairs of stimuli and, say, certain attributes of the local contexts within which they are encoded. The encoding of the processing carried out on the stimuli would incorporate the relatedness between the paired items, whereas the encoding of local contextual attributes would highlight their differences. On the basis of the latter components, subjects could discriminate similar traces and infer frequency of occurrence, spacing, and so on. These contextual components could also be responsible for the spacing effect that was found within the different-question condition of the experiments reported here. The point made by the results of Hintzman and his colleagues is that these contextual components are not efficient 
"benchmarks" unless they are incorporated into the encoding of related events.

What is advocated here, then, as an explanation of the spacing effect is a levels-of-processing hypothesis that is a modification of the component-levels theory. The notion of increasing variability of certain components over spacing is retained mainly as an explanation of the discriminability of traces of repeated items. However, crucial emphasis is placed upon the similarity of processing of repeated items at fairly long lags. The depth of processing necessary to attain a successful reconstruction increases over lag and is the major source of the spacing effect.

\section{REFERENCES}

Craik, F. I. M., \& Lockhart, R. S. Levels of processing: A framework for memory research. Journal of Verbal Learning and Verbal Behavior, 1972, 11, 671-684.

Craik, F. I. M., \& Tulving, E. Depth of processing and the retention of words in episodic memory. Journal of Experimental Psychology: General, 1975, 104, 268-294.

D'Agostino, P. R., \& DeRemer, P. Repetition effects as a function of rehearsal and encoding variability. Journal of Verbal Learning and Verbal Behavior, 1973, 12, 108-113.

Flexser, A. J., \& Bower, G. H. Further evidence regarding instructional effects on frequency judgments. Bulletin of the Psychonomic Society, 1975, 6, 321-324.

Gartman, L. M., \& Johnson, N. F. Massed versus distributed repetition of homographs: A test of the differential-encoding hypothesis. Journal of Verbal Learning and Verbal Behavior, 1972, 11, 801-808.

Glenbe RG, A. Monotonic and nonmonotonic lag effects in pairedassociate and recognition memory paradigms. Journal of Verbal Learning and Verbal Behavior, 1976, 15, 1-16.

Glenberg, A. Component-levels theory of the effects of spacing of repetitions on recall and recognition. Memory \& Cognition, $1979,7,95-112$.

Hintzman, D. L. Theoretical implications of the spacing effect. In R. L. Solso (Ed.), Theories in cognitive psychology: The Loyola symposium. Potomac, Md: Erlbaum, 1974.

Hintzman, D. L. Repetition and memory. In G. H. Bower (Ed.), The psychology of learning and motivation (Vol. 10). New York: Academic Press, 1976.

Hintzman, D. L., \& Block, R. A. Memory for the spacing of repetitions. Journal of Experimental Psychology, 1973, 99, 70-74.

Hintzman, D. L., \& Rogers, M. K. Spacing effects in picture memory. Memory \& Cognition, 1973, 1, 430-434.
Hintzman, D. L., \& Stern, L. D. Contextual variability and memory for frequency. Journal of Experimental Psychology: Human Learning and Memory, 1978, 4, 539-549.

Hintzman, D. L., Summers, J. J., \& Block, R. A. Spacing judgments as an index of study-phase retrieval. Journal of Experimental Psychology: Human Learning and Memory, 1975, $1,21-40$.

Howell, W. C. Representation of frequency in memory. Psychological Bulletin, 1973, 80, 44-53.

J ACOBY, L. L. The role of mental contiguity in memory: Registration and retrieval effects. Journal of Verbal Learning and Verbal Behavior, 1974, 13, 483-496.

Johnston, W. A., Coots, J. H., \& Flickinger, R. G. Controlled semantic encoding and the effect of repetition lag on free recall. Journal of Verbal Learning and Verbal Behavior, 1972, 11, 784-788.

Johnston, W. A., \& UhL, C. N. The contributions of encoding effort and variability to the spacing effect on free recall. Journal of Experimental Psychology: Human Learning and Memory, 1976, 2, 153-160.

Lockhart, R. S., Craik, F. I. M., \& Jacoby, L. L. Depth of processing in recognition and recall: Some aspects of a general memory system. In J. Brown (Ed.), Recall and recognition. London: Wiley, 1976.

Madigan, S. A. Intraserial repetition and coding processes in free recall. Journal of Verbal Learning and Verbal Behavior, $1969,8,828-835$.

Melton, A. W. Repetition and retrieval from memory. Science, 1967, 158, 532.

Rose, R. J., \& Rowe, E. J. Effects of orienting task and spacing of repetitions on frequency judgments. Journal of Experimental Psychology: Human Learning and Memory, 1976, 2, 142-152.

Rowe, E. J. Context effects in judgment of frequency. Bulletin of the Psychonomic Society, 1973, 2, 231-232. (a)

Rowe, E. J. Frequency judgments and recognition of homonyms. Journal of Verbal Learning and Verbal Behavior, 1973, 12, 440-447. (b)

Rowe, E. J., \& Rose, R. J. Effects of orienting task, spacing of repetitions, and list context on judgments of frequency. Memory \& Cognition, 1977, 5, 505-512.

THios, S. J. Memory for words in repeated sentences. Journal of Verbal Learning and Verbal Behavior, 1972, 11, 789-793.

Thorndike, E. L., \& LoRge, I. The teacher's word book of 30,000 words. New York: Columbia University, Teacher's College, 1944.

Tulving, E. Ecphoric processes in recall and recognition. In J. Brown (Ed.), Recall and recognition. London: Wiley, 1976.

Underwood, B. J., Zimmerman, J., \& Freund, J. S. Retention of frequency information with observations on recognition and recall. Journal of Experimental Psychology, 1971, 87, 149-162.

(Received for publication April 5, 1979; revision accepted October $31,1979$. 